\title{
Review
}

\section{Colorectal Awareness in Saudi Arabia: A Systematic Review of Cross-Sectional Studies}

\author{
Omar Alshaer ${ }^{1}$, Abdullah Binobaid ${ }^{1}$, Mohammed Alqahtani ${ }^{2}$, Mohammed Alabdullah ${ }^{3}$, Amal Assiri², Huda \\ AlGossadi $^{4}$, Azizah Alreshi ${ }^{5}$, Mohammed Alsayed ${ }^{6}$, Reem Alghamdi ${ }^{7}$, Sarah Alhussain ${ }^{8}$, Buthaynah Alghasham ${ }^{9}$ \\ ${ }^{1}$ Department of Internal Medicine, Security Forces Hospital, Riyadh, Saudi Arabia \\ ${ }^{2}$ Department of Internal Medicine, King Fahad Armed Forces Hospital, Asir, Saudi Arabia \\ ${ }^{3}$ College of Medicine, Medical University of Lodz, Lodz, Poland \\ ${ }^{4}$ Department of General Surgery, King Fahad Armed Forces Hospital, Asir, Saudi Arabia \\ ${ }^{5}$ Department of Internal Medicine, Alnoor Specialist Hospital, Mecca, Saudi Arabia \\ ${ }^{6}$ Department of Internal Medicine, Al Qunfudah General Hospital, Al Qunfudah, Saudi Arabia \\ ${ }^{7}$ College of Medicine, King Abdulaziz University, Jeddah, Saudi Arabia \\ ${ }^{8}$ College of Medicine, King Khalid University, Abha, Saudi Arabia \\ ${ }^{9}$ King Khalid Primary Hospital Health Care, Ministry of Health, Qassim, Saudi Arabia
}

Correspondence should be addressed to Omar Alshaer, Department of Internal Medicine, Security Forces Hospital, Riyadh, Saudi Arabia Email: oalshaer@sfh.med.sa

Received 19 June 2021; Revised 22 June 2021; Accepted 23 June 2021; Published 25 June 2021

Copyright (C) 2021 Alshaer et al. This is an open access article distributed under the Creative Commons Attribution License, which permits unrestricted use, distribution, and reproduction in any medium, provided the original work is properly cited.

\begin{abstract}
Colorectal cancer is the second most common cancer in Saudi Arabia with estimated prevalence rates for males and females at $9.9 \%$ and $6.4 \%$, respectively. It is also the most common cancer in males and the third most common cancer in females in the kingdom. In general, the evidence regarding knowledge and awareness of colorectal cancer in Saudi Arabia is conflicting among different studies. Accordingly, we conducted a systematic review that aims to formulate strong evidence regarding the awareness and knowledge of colorectal cancer in Saudi Arabia. A systematic search was conducted through several databases to find the relevant articles. A total of 19 cross-sectional investigations were found fit in our inclusion criteria and were included in the final data. The sample size was hugely variable among these studies, ranging between 127 and 5,720, with a total of 18,525 included participants. Most studies recruited participants from the general population, however, some studies recruited school teachers, medical and university students as well as healthcare workers. Results show that the prevalence of poor awareness levels and inadequate knowledge of colorectal cancer in Saudi Arabia is high. Although the levels of knowledge among healthcare workers was high, most of them did not follow advisable screening protocols for colorectal cancer. Nationwide programs should be inaugurated to increase the level of awareness and knowledge among the Saudi population and enhance the prognosis and outcomes of colorectal cancer across the Kingdom.
\end{abstract}

Keywords: colorectal cancer, saudi arabia, knowledge, awareness, epidemiology, systematic review. 
Journal of Healthcare Sciences

\section{Introduction}

Colorectal cancer (CRC) is the fourth worldwide leading cause of death and the third most common cancer globally. Projections in dicate that the prevalence of CRC will increase by $60 \%$ by 2030 . Evidence from low socioeconomic countries also shows that the prevalence rates are higher in these countries and that the burden rapidly rises due to the growing attitudes toward western lifestyles in these countries (1). Estimates from the Kingdom of Saudi Arabia show that CRC is the second most common cancer, with estimated prevalence rates for males and females at $9.9 \%$ and $6.4 \%$, respectively. It is also the most common malignancy in males and the third most common cancer in females in the kingdom (2). Moreover, previous investigations have reported that the rate of CRC doubled between 2015 and 2001, as 9.6 per 100,000 patients were diagnosed with CRC by 2015 $(3,4)$.

It has also been previously reported that the survival rate from CRC in Saudi Arabia is lower than the previously reported global rates, which may be attributable to the late presentation of the affected patients $(5,6)$. Patients suffering from CRC usually present with non-specific local and systemic symptoms including fatigue and loss of appetite and weight, and the condition can be mistaken for the symptoms of irritable bowel syndrome (7-9). Therefore, an early diagnosis of the modality is encouraged to achieve better outcomes. Public and routine screening for $\mathrm{CRC}$ is recommended to significantly enhance the survival rates among these patients. Screening is also recommended for high-risk groups, including patients with a previous history of polyps and CRC $(10,11)$.

It has also been demonstrated that screening for $\mathrm{CRC}$ is usually favorable and enhances the outcomes of patients with CRC due to the long interval between the early and late stages of the condition $(12,13)$. Although routine screening is encouraged for patients before the age of 50 , it has been reported that patients in Saudi Arabia tend to present late when suffering from conditions that often pose a poor prognosis $(3,14,15)$.
This indicates the need to raise the awareness within the general population regarding $\mathrm{CRC}$ to enhance the quality of care of CRC and achieve better outcomes.

Although it has been previously reported that Saudi individuals may have enough information about $\mathrm{CRC}$ screening, it was also reported that the many factors including reduced knowledge, fear of the screening modalities, and embarrassment were associated with a reduced frequency in seeking CRC screening (10). In general, evidence regarding knowledge and awareness of CRC in Saudi Arabia is conflicting among the different studies. As such, we conducted this systematic review with the aim to formulate strong evidence regarding the awareness and knowledge of CRC in Saudi Arabia.

\section{Methods}

\section{Outcomes, inclusion, and exclusion criteria}

Our present systematic review aims to provide evidence about the current knowledge and awareness levels of CRC in Saudi Arabia, according to the previously published cross-sectional investigations within the Kingdom.

We included studies that were: 1) cross-sectional in design, 2) recruited patients within Saudi Arabia, including saudis and non-saudis, on the condition that it was conducted within a specific region across the Kingdom, 3) human studies, and 4) reported the prevalence of knowledge and awareness levels among such populations or estimated the knowledge and awareness total values using any previously validated assessment tool. Studies were excluded if they: 1) were not cross-sectional, 2) were not conducted in Saudi Arabia, 3) did not recruit patients with CRC and did not investigate the level of knowledge and awareness within the recruited populations, and 4) were not original in design, including thesis, commentary, letters, editorials, or were not human studies. 
Journal of Healthcare Sciences

\section{Search strategy and screening}

All of the following steps were undertaken based on the recommendations from the Preferred Reporting Items for Systematic Reviews and Meta-Analyses (PRISMA) guidelines (16). Many databases were searched to find the relevant articles, in addition to manually searching the references of these databases and the relevant reviews to find any potentially missed articles during the electronic database searching and screening of exported results.

The following search term was used for PubMed: (("knowledge" OR "attitude" OR "practice" OR "aware") AND ("colorectal cancer" OR "colon cancer" OR "CRC") AND ("Saudi Arabia" OR "Saudi")). The term was then modified per each database in accordance with their guidelines, in order to achieve the best result. We searched Web of Science, Scopus, Google Scholar, Embase, Cochrane Library, and the Virtual Health Library $(17,18)$.

Title and abstract screening and full-text screening was then performed to identify the relevant articles according to the inclusion and exclusion criteria, which were designed to find the data relevant to our outcomes. Each step was adequately explained to all members and Endnote and Microsoft Excel programs were used to gather all the search results and to facilitate the process of screening, in addition to the easy identification of duplicates among the different databases.

\section{Data extraction and quality assessment}

After adequate screening to ensure that all the relevant studies were included, we moved to the next step by inaugurating data extraction, which was done by all of the study members. First, a senior member was asked to design a suitable sheet that suited all the included articles to be used for easy extraction of the relevant data. Then, piloting of the sheet was undertaken to determine the comprehensiveness. The sheet consisted of three different parts, including one part for the baseline characteristics and methodology of the included investigations, such as the relevant data about the authors, study design, data collection, sample size, region, gender and age of the included population.
The second part consisted of the intended outcomes, including the levels of awareness and knowledge of CRC, knowledge of screening, symptoms and risk factors. However, reporting on these outcomes was inconsistent among the included studies, and therefore, our extraction of these variables was flexible where we included all the relevant information as far as possible. The third part included the domains and results of the quality assessment of the included investigations.

To assess the risk of bias among the included crosssectional studies, the modified Newcastle-Ottawa Scale (NOS) for cross-sectional studies (19) was utilized and explained for all members in order to properly assess the quality of the included investigations. Three principle domains were used to formulate this tool, including the assessment and compatibility, quality of methods, and outcomes. Each of these steps was marked by a specific score, to grade and classify each article by their estimated quality and risk of bias as: excellent, nonsatisfactory, good or satisfactory.

The lowest score was 0 , referring to low quality and a high risk of bias, and the largest score was 10 , referring to high quality and a low risk of bias. All of the extraction steps were performed by at least two blind reviewers and under the supervision of the senior member, and all discussed all the conflicts and differences until they have finally agreed on a single decision, based on the inclusion and exclusion criteria.

\section{Results}

\section{Search results}

After searching all of the databases, we managed to retrieve a total of 592 relevant citations that would suit our outcomes, of which 148 duplicates were removed by the Endnote program. This left only 444 citation for title/abstract screening, after which only 39 articles were eligible for full-text downloading and screening. Ultimately, 16 articles were identified, in addition to 3 other articles that were gathered via manual search, leaving us with a final list of 19 relevant articles. These steps are summarized in Figure 1. 
Figure 1. Flow diagram of the included articles according to the steps of PRISMA.

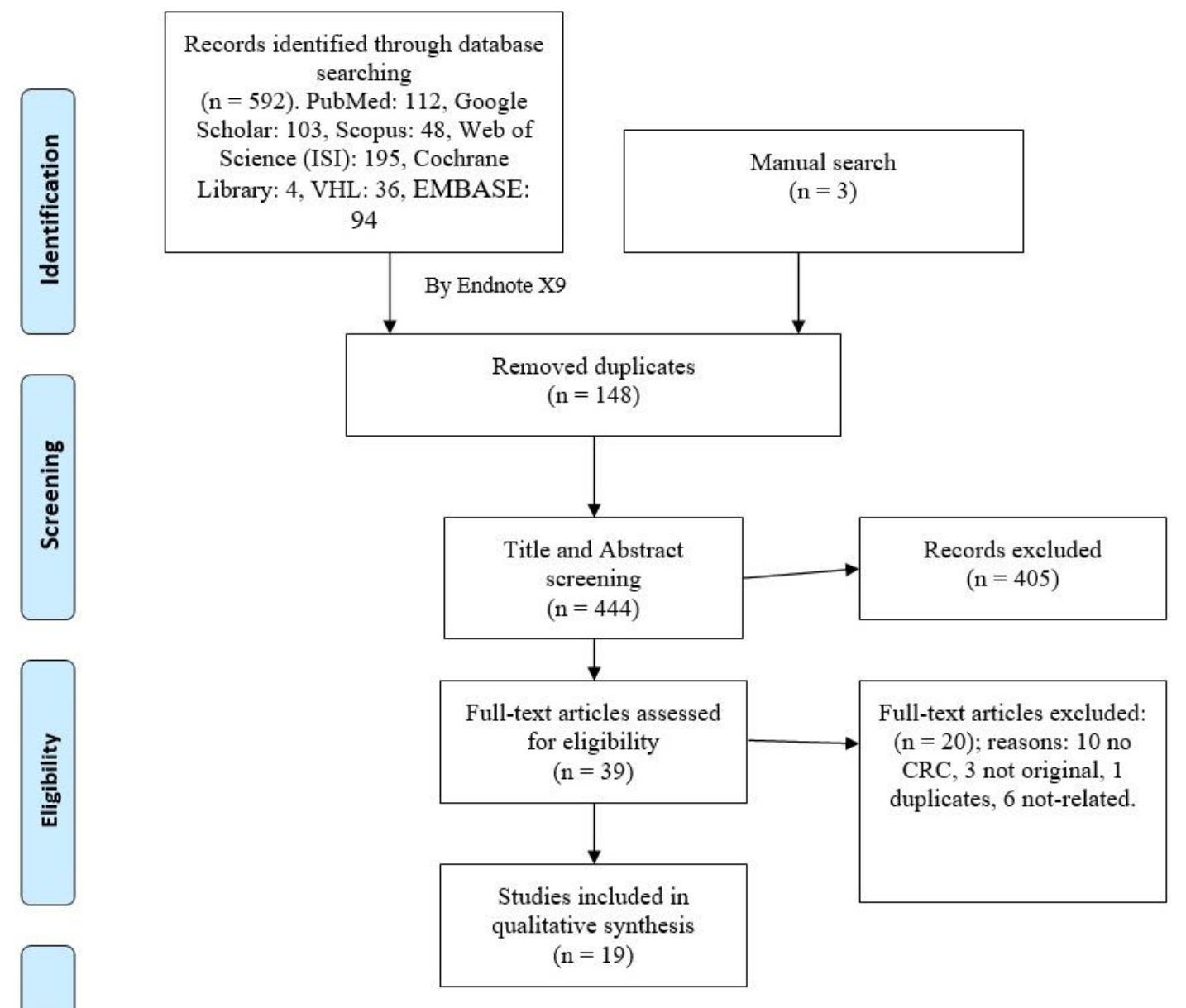

\section{Risk of bias}

Our assessment of the quality of the included studies showed acceptable results with variable degrees of bias in the included studies in the different assessed domains of the mNOS tool and is fully presented in Table 1.
In brief, 11 articles were marked 'good' with total scores of 7 or 8 , while the rest of the articles were marked 'satisfactory' with total scores of 5 or 6 . This indicates that the risk of bias is relatively acceptable among these investigations. 


\section{Journal of Healthcare Sciences}

Table 1.Detailed results of the quality of the included studies according to the modified Newcastle-Ottawa scale (mNOS) for cross-sectional studies

\begin{tabular}{|c|c|c|c|c|c|c|c|c|c|c|}
\hline \multirow[b]{2}{*}{ Author } & \multirow[b]{2}{*}{ Year } & \multicolumn{4}{|c|}{ Selection } & \multirow{2}{*}{$\begin{array}{c}\text { Comparability } \\
\\
\text { The Subjects in } \\
\text { Different } \\
\text { Outcome Groups } \\
\text { are Comparable }\end{array}$} & \multicolumn{2}{|c|}{ Outcome } & \multirow[b]{2}{*}{$\begin{array}{l}\text { Total } \\
\text { score }\end{array}$} & \multirow[b]{2}{*}{ Quality } \\
\hline & & $\begin{array}{c}\text { Representativenes } \\
\text { s of the Sample }\end{array}$ & $\begin{array}{l}\text { Sample } \\
\text { size }\end{array}$ & $\begin{array}{c}\text { Non } \\
\text { Respondents }\end{array}$ & $\begin{array}{c}\text { Ascertainment } \\
\text { of the Exposure }\end{array}$ & & $\begin{array}{l}\text { Assessment } \\
\text { of outcome }\end{array}$ & $\begin{array}{c}\text { Statistical } \\
\text { analysis }\end{array}$ & & \\
\hline Ahmed et al.(20) & 2020 & + & + & + & + & & + & & 5 & Satisfactory \\
\hline Al-Hajeili et al.(21) & 2019 & + & + & + & + & + & + & + & 7 & Good \\
\hline Alhuzaim et al.(10) & 2020 & + & + & + & + & ++ & + & + & 8 & Good \\
\hline Alkhayyat et al.(22) & 2021 & + & + & + & & + & + & + & 6 & Satisfactory \\
\hline Almadi et al.(23) & 2019 & + & + & & + & + & + & + & 6 & Satisfactory \\
\hline Almadi et al.(24) & 2015 & + & + & + & ++ & + & + & + & 8 & Good \\
\hline Almutairi et al.(25) & 2018 & + & + & + & ++ & + & + & + & 8 & Good \\
\hline Alotaibi et al.(26) & 2020 & + & + & + & + & ++ & + & + & 8 & Good \\
\hline Alsayed et al.(27) & 2019 & + & + & + & + & + & & + & 5 & Satisfactory \\
\hline $\begin{array}{l}\text { Alshammari } \\
\text { et al.(28) }\end{array}$ & 2020 & + & + & + & + & + & + & + & 7 & Good \\
\hline Al-Sharif et al.(29) & 2018 & + & + & + & + & + & + & + & 7 & Good \\
\hline Al-Thafar et al.(30) & 2017 & + & + & + & + & + & + & + & 7 & Good \\
\hline Althobaiti et al.(31) & 2019 & + & + & & + & + & + & + & 6 & Satisfactory \\
\hline Demyati(32) & 2014 & + & + & + & + & + & & + & 6 & Satisfactory \\
\hline Galal et al.(33) & 2016 & + & + & + & + & + & + & + & 7 & Good \\
\hline Imran et al.(34) & 2016 & + & + & + & + & + & + & + & 7 & Good \\
\hline Khayyat et al.(35) & 2014 & + & + & + & + & + & & + & 6 & Satisfactory \\
\hline Mosli et al. (36) & 2017 & + & + & & + & + & & + & 5 & Satisfactory \\
\hline Zubaidi et al. (37) & 2015 & + & + & + & ++ & + & + & + & 8 & Good \\
\hline
\end{tabular}


Journal of Healthcare Sciences

\section{Baseline characteristics}

We managed to include 19 cross-sectional studies across the different regions within the Kingdom of Saudi Arabia. The sample size was hugely variable among these studies, ranging between 127 and 5,720, with a total of 18,525 included participants. The age ranges and male/female ratio were also variable and can be seen in Supplementary Table 1. Jeddah and Riyadh were the most frequent regions from where participants were recruited, followed by other regions including AlHassa or Al-Ahsa, Asir, Dammam, Mekkah, Almadinah and Albaha. This was in addition to the nationwide investigations that did not limit their recruitment target to a certain region. Most studies recruited participants from the general population, however, some studies recruited school teachers, and medical and university students as well as healthcare workers. Details of these characteristics, in addition to a summary of the outcomes and results of each investigation are presented in Supplementary Table 1.

\section{Discussion}

In Riyadh, an investigation which was conducted in 2021 by Alshammari et al. (28) reported that among their 231 participants, $47 \%$ knew about cancer screening, while another $45 \%$ and $24.4 \%$ knew that colonoscopy and that occult blood in the stools can be used for screening and early detection of CRC, respectively. Up to $50 \%$ of the participants knew about the risk factors of CRC and only investigation in Riyadh by Almadi et al. (23) that approximately 58\% and 53\% of them had poor included 5,720 participants in their survey reported that knowledge about the screening modalities for CRC and the mean CRC knowledge score among their study the associated risk factors, respectively. The authors also participants was 11.05 and that $73 \%$ of them were reported that having good attitudes towards CRC willing to undergo CRC screening. However, they also screening and higher educational levels were reported that a significant gap between the knowledge significantly associated with elevated knowledge scores. and practice of screening was found among their study Conversely, Imran et al. (34) included 525 university participants. Demyati et al. (32) also investigated CRC in students from Jeddah to find that $82.3 \%$ of them were Riyadh city to assess the level of knowledge among aware of CRC and 56.8\% reported that CRC could be family physicians in the area. They reported that adequately managed. Moreover, up to $60 \%$ of the approximately $95 \%$ reported that CRC screening is an participants reported that they were aware of the effective modality. However, approximately $56 \%$ of the symptoms and risk factors of CRC. The authors also participants did not participate in CRC screening and the reported that the knowledge levels between the nonknowledge scores were higher in physicians that medical and medical students were statistically participated in CRC screening than those who did not. significant. This indicates that medical students may Almutairi et al. (25) also reported that the level of have better knowledge and awareness of the disease. knowledge in Riyadh among their 256 adult participants was low, and they reported that most of the study

Page 72 
However, the overall levels of the whole population were not favorable. In Jeddah, Al-Hajeili et al. (21) reported that among 422 participants in their crosssectional analysis, gender was a significant predictor for knowledge levels, and having a previous family history of CRC and the level of education were the only significant predictors for awareness of CRC screening. Fearing the results, absence of the clinical symptoms and having a fear of the screening process were the most commonly reported factors for not undergoing CRC screening. This was also supported by another investigation in the Al-Hassa region which showed that fear of the screening procedures was the most commonly reported reason for an unwillingness to participate in the CRC screening campaigns (33).

In contrast, in the study by Almadi et al. (24) in Riyadh, the authors reported that knowing that a colonoscopy was the used screening modality was significantly associated with a higher willingness to undergo screening, although stool-based screening modalities were not. Moreover, the study that was conducted in AlHassa also reported that from the healthcare physicians perspectives, a lack of awareness and symptoms of CRC, in addition to having a fear of CRC screening procedures, were the most common factors that were associated with a reduced willingness to attend CRC screening (33). Khayyat et al. (35) also reported that among 321 study participants from Jeddah, only around $37 \%$ of them had previously heard of CRC screening, and approximately $55 \%$ of them expressed the desire to undergo colonoscopy sigmoidoscopy for CRC. Moreover, they reported that educational levels, but not age or gender, were significantly associated with the awareness levels of the participants. Mosli et al. (36) reported that among 127 primary health physicians who participated in their local survey from Jeddah, 95\% reported that CRC screening is an effective modality, but only $45 \%$ participated in such campaigns. Moreover, they reported that being male, and holding a bachelor's degree or less were significantly associated with reduced frequencies of recommending CRC screening. Comparing these results to the findings that were previously reported in Riyadh (32) indicates that although the healthcare workers in Saudi Arabia had adequate knowledge of CRC screening, they did not adequately stick to the screening and management protocols and recommendations.
Among 367 school teachers in Al-Ahsa, Al-Thafar et al. (33) reported that $42 \%$ and $39 \%$ of the female and male participants were not aware of the CRC screening tests, respectively, and higher levels of awareness were associated with higher educational levels. Moreover, the authors reported that most of the participants did not have enough knowledge about CRC or the associated risk factors. In the Asir region, Al-Sharif et al. (29) reported that among 1,209 participants in their crosssectional investigation, up to $71.6 \%$ knew what a colon or rectum is, while around one-third of them reported the right function for the colon and rectum. Moreover, approximately $22 \%$ and $23 \%$ of the participants reported that they knew the correct timing of CRC screenings and the correct incidence, respectively, and being male with a lower level of education was significantly associated with lower awareness and knowledge levels. This indicates the significantly low knowledge and awareness levels of CRC in their population. In the Al-Hassa region, Galal et al. (33) reported that the level of knowledge among the study participants, which included participants from the general population and family physicians, was low among over two-thirds of the group. Screening for CRC was significantly lower in patients that were unmarried, female, had a lower level of education and with no previous family history of CRC. In Madinah, it was also reported that the levels of knowledge and awareness among the general population were low regarding CRC (27).

Another investigation was also conducted in Saudi Arabia but was confined to a certain region, and the authors reported that among 128 study participants, 57\% knew what the colon and its functions are, and approximately 59\% reported that they would undergo CRC screening in the event of relevant symptoms, and only $9 \%$ and $4 \%$ reported that they believed that inflammatory bowel diseases and radiotherapy were significant risk factors for CRC development (20). Alkhayyat et al. (22) managed to include 4,090 individuals from all over the Kingdom of Saudi Arabia and found that approximately $44 \%$ of the study participants reported that unintentional weight loss was the main significant sign associated with CRC. Moreover, approximately $61 \%$ of the study participants were not aware of the CRC screening methods. A further nationwide investigation was conducted by Alotaibi et al. (26) that reported that the 521 included 
Journal of Healthcare Sciences

participants, approximately $71 \%$ had the desire to be screened for CRC. However, the levels of knowledge and awareness were low in approximately half of the participants in this study, as assessed by low knowledge of the symptoms and risk factors for developing CRC. Another nationwide investigation was also conducted in six regions in Saudi Arabia including Jeddah, Mekkah, Riyadh, Dammam, Albaha and AlAhsa. The study managed to recruit 925 participants, of whom $95 \%$ were adequately aware of CRC, and more than $50 \%$ were knowledgeable of the disease. However, not many of them participated in the screening programs (10).

Our findings may be limited by the heterogeneity among the included studies when reporting the outcomes and unification of the methodology. Moreover, the limited sample size also carries another limitation across many of the included studies, and many regions across the Kingdom were not investigated as most of the published investigations were limited to certain regions such as Riyadh and Jeddah. Although the results seem to be conclusive among most of the included investigations, further nationwide large scale research is needed.

\section{Conclusion}

Results show that the prevalence of poor awareness levels and inadequate knowledge of CRC in Saudi Arabia is high. Although the levels of knowledge among the healthcare workers were high, most of them did not follow advisable screening protocols for CRC. Nationwide programs should be inaugurated to increase the level of awareness and knowledge among the Saudi population and to enhance the prognosis and outcomes of CRC across the Kingdom.

\section{Disclosure}

\section{Statement:}

The authors declare that there is no conflict of interest.

\section{Funding:}

None

\section{Ethical Consideration:}

This study was approved by Security Forces Hospital Instituitonal Review Board (IRB) with approval number H-01-R-069.

\section{References}

1. Arnold M, Sierra MS, Laversanne M, Soerjomataram I, Jemal A, Bray F. Global patterns and trends in colorectal cancer incidence and mortality. Gut. 2017;66(4):683-91.

2. Alqahtani WS, Almufareh NA, Domiaty DM, Albasher G, Alduwish MA, Alkhalaf $\mathrm{H}$, et al. Epidemiology of cancer in Saudi Arabia thru 2010-2019: a systematic review with constrained meta-analysis. AIMS Public Health. 2020;7(3):679-96.

3. Alsanea N, Abduljabbar AS, Alhomoud S, Ashari LH, Hibbert D, Bazarbashi S. Colorectal cancer in Saudi Arabia: incidence, survival, demographics and implications for national policies. Annals of Saudi medicine. 2015;35(3):196-202.

4. Al-Zalabani A. Preventability of Colorectal Cancer in Saudi Arabia: Fraction of Cases Attributable to Modifiable Risk Factors in 20152040. International journal of environmental research and public health. 2020;17(1).

5. Al-Ahwal MS, Shafik YH, Al-Ahwal HM. First national survival data for colorectal cancer among Saudis between 1994 and 2004: what's next? BMC public health. 2013;13:73.

6. Forbes LJ, Simon AE, Warburton F, Boniface D, Brain KE, Dessaix A, et al. Differences in cancer awareness and beliefs between Australia, Canada, Denmark, Norway, Sweden and the UK (the International Cancer Benchmarking Partnership): do they contribute to differences in cancer survival? British journal of cancer. 2013;108(2):292-300.

7. Hansen PL, Hjertholm P, Vedsted P. Increased diagnostic activity in general practice during the year preceding colorectal cancer diagnosis. International journal of cancer. 2015;137(3):61524.

8. Rasmussen S, Larsen PV, Søndergaard J, Elnegaard S, Svendsen RP, Jarbøl DE. Specific and non-specific symptoms of colorectal cancer and contact to general practice. Family practice. 2015;32(4):387-94. 
9. Oberoi DV, Jiwa M, McManus A, Hodder R. Men's help-seeking behavior with regards to lower bowel symptoms. American journal of health behavior. 2015;39(2):212-21.

10. Alhuzaim W, Alosaimi M, Almesfer AM, Al Shahrani NM, Alali AH, Alibrahim KIF, et al. Saudi Patients' Knowledge, Behavior, Beliefs, Self-Efficacy and Barriers Regarding Colorectal Cancer Screening. International Journal of Pharmaceutical Research \& Allied Sciences. 2020;9(1).

11. Bhatt DB, Emuakhagbon V-S. Current trends in colorectal cancer screening. Current Colorectal Cancer Reports. 2019;15(2):45-52.

12. Smith RA, Manassaram-Baptiste D, Brooks D, Doroshenk M, Fedewa S, Saslow D, et al. Cancer screening in the United States, 2015: a review of current American cancer society guidelines and current issues in cancer screening. CA: a cancer journal for clinicians. 2015;65(1):30-54.

13. Mack LA, Stuart H, Temple WJ. Survey of colorectal cancer screening practices in a large Canadian urban centre. Canadian journal of surgery Journal canadien de chirurgie. 2004;47(3):189-94.

14. Veen T, Stormark K, Nedrebø BS, Berg M, Søreide JA, Kørner H, et al. Long-Term FollowUp and Survivorship After Completing Systematic Surveillance in Stage I-III Colorectal Cancer: Who Is Still at Risk? J Gastrointest Cancer. 2015;46(3):259-66.

15. Aljebreen AM. Clinico-pathological patterns of colorectal cancer in Saudi Arabia: younger with an advanced stage presentation. Saudi journal of gastroenterology : official journal of the Saudi Gastroenterology Association. 2007;13(2):84-7.

16. Liberati A, Altman DG, Tetzlaff J, Mulrow C, Gøtzsche PC, Ioannidis JPA, et al. The PRISMA statement for reporting systematic reviews and meta-analyses of studies that evaluate healthcare interventions: explanation and elaboration. BMJ. 2009;339:b2700.
17. Hashan MR, Ghozy S, El-Qushayri AE, Pial RH, Hossain MA, Al Kibria GM. Association of dengue disease severity and blood group: A systematic review and meta-analysis. Rev Med Virol. 2021;31(1):1-9.

18. El-Qushayri AE, Ghozy S, Abbas AS, Dibas M, Dahy A, Mahmoud AR, et al. Hyperimmunoglobulin therapy for the prevention and treatment of congenital cytomegalovirus: a systematic review and meta-analysis. Expert Rev Anti Infect Ther. 2020:1-9.

19. Newcastle- Ottawa: Quality assessment scale adapted for cross-sectional studies.

20. Ahmed NJ, Alrashidi M. Saudi Public Awareness Regarding Colon Cancer. Journal of Pharmaceutical Research International. 2020:23-8

21. Al-Hajeili M, Abdulwassi H, Alshadadi F, Alqurashi L, Idriss M, Halawani L. Assessing knowledge on preventive colorectal cancer screening in Saudi Arabia: A cross-sectional study. J Family Med Prim Care. 2019;8:3140

22. Alkhayyat S, Khojah M, AlJehan M, Allali D, Tayeb A, Albukhari S, et al. Awareness of Colorectal Cancer in Saudi Arabia: CrossSectional Study. Pharmacophore. 2021;12:38-43.

23. Almadi MA, Alghamdi F. The gap between knowledge and undergoing colorectal cancer screening using the Health Belief Model: A national survey. Saudi journal of gastroenterology : official journal of the Saudi Gastroenterology Association. 2019;25(1):27-39.

24. Almadi MA, Mosli MH, Bohlega MS, Al Essa MA, AlDohan MS, Alabdallatif TA, et al. Effect of public knowledge, attitudes, and behavior on willingness to undergo colorectal cancer screening using the health belief model. Saudi journal of gastroenterology : official journal of the Saudi Gastroenterology Association. 2015;21(2):71-7. 


\section{Journal of Healthcare Sciences}

25. Almutairi KM, Alonazi WB, Alodhayani A, Vinluan JM, Ahmad M, Alhurishi SA, et al. A Cross-Sectional Assessment of Literacy and Awareness, Attitudes, and Beliefs About Colorectal Cancer and Its Screening in Riyadh Region. Journal of cancer education : the official journal of the American Association for Cancer Education. 2018;33(3):660-7.

26. Alotaibi N, Mujtaba M, Alshammari N. Knowledge, Attitudes and Awareness about Colorectal Cancer in the Kingdom of Saudi Arabia: A Cross Sectional Study. Journal of Young Pharmacists. 2020;12:266-70.

27. Alsayed MA, Surrati AM, Altaifi JA, Alharbi AH, Alfouti RO, Alremaithi SM. Public Awareness of Colon Cancer Symptoms, Risk Factor, and Screening at Madinah-KSA. International Journal of Pharmaceutical Research \& Allied Sciences. $2019 ; 8(1)$.

28. Alshammari SA, Alenazi HA, Alshammari HS. Knowledge, attitude and practice towards early screening of colorectal cancer in Riyadh. J Family Med Prim Care. 2020;9(5):2273-80.

29. Al-Sharif M, Fayi K, Alobaidi A, Alshamrani B. Awareness of colorectal cancer among public in Asir region. J Family Med Prim Care. 2018;7:87.

30. Al-Thafar AK, Al-Naim AF, Albges DS, Boqursain SK, Aldhafar AS, Ghreiz SM, et al. Knowledge Attitude and Practice of Colorectal Cancer among School Teachers in Al-Ahsa Saudi Arabia. Asian Pacific journal of cancer prevention : APJCP. 2017;18(10):2771-4.

31. Althobaiti A, Jradi H. Knowledge, attitude, and perceived barriers regarding colorectal cancer screening practices and risk factors among medical students in Saudi Arabia. BMC medical education. 2019;19(1):421.
32. Demyati E. Knowledge, Attitude, Practice, and Perceived Barriers of Colorectal Cancer Screening among Family Physicians in National Guard Health Affairs, Riyadh. International journal of family medicine. 2014;2014:457354.

33. Galal YS, Amin TT, Alarfaj AK, Almulhim AA, Aljughaiman AA, Almulla AK, et al. Colon Cancer among Older Saudis: Awareness of Risk Factors and Early Signs, and Perceived Barriers to Screening. Asian Pacific journal of cancer prevention : APJCP. 2016;17(4):1837-46.

34. Imran M, Sayedalamin Z, Alsulami SS, Atta M, Baig M. Knowledge and Awareness of Colorectal Cancer among Undergraduate Students at King Abdulaziz University, Jeddah, Saudi Arabia: a Survey-Based Study. Asian Pacific journal of cancer prevention : APJCP. 2016;17(5):2479-83.

35. Khayyat YM, Ibrahim EM. Public awareness of colon cancer screening among the general population: A study from the Western Region of Saudi Arabia. Qatar medical journal. 2014;2014(1):17-24.

36. Mosli M, Alnahdi Y, Alghamdi A, Baabdullah M, Hadadi A, Khateery K, et al. Knowledge, attitude, and practices of primary health care physicians toward colorectal cancer screening. Saudi journal of gastroenterology : official journal of the Saudi Gastroenterology Association. 2017;23(6):330-6.

37. Alotaibi N, Mujtaba M, Alshammari N. Knowledge, Attitudes and Awareness about Colorectal Cancer in the Kingdom of Saudi Arabia: A Cross Sectional Study. Journal of Young Pharmacists. 2020;12:266-70. 